\title{
Comunidades de aprendizaje: una perspectiva de la educación inclusiva
}

\author{
Martha Luz Lanza Elvir ${ }^{1}$ \\ William Oswaldo Flores López ${ }^{2}$
}

\section{Resumen}

G este artículo se analizó la metodologia de comunidades de aprendizaje desde una perspectiva de la Eeducación inclusiva. Se trata de un estudio cualitativo con un enfoque hermenéutico hacia los contenidos de libros de textos, artículos científicos, artículos de prensa, leyes, curriculos, programas de formación y normas institucionales. Los resultados certifican que la metodología comunidades de aprendizajes juega una papel importante en la educación inclusiva, porque toma en cuenta: el diálogo y la acción comunicativa; aprendizaje dialógico; modelo dialógico; grupos interactivos y tertulias literarias, permitiendo mejorar la convivencia, el desarrollo de capacidades, de diálogo y critica para una construcción de una sociedad igualitaria, multicultural y solidaria. Por lo tanto, las comunidades de aprendizaje ayudan a la superación de las desigualdades y riesgo de exclusión social, así como la tendencia al fracaso del estudiantado.

Palabras clave: Comunidades de aprendizaje; educación inclusiva; aprendizaje dialógico; desigualdades; riesgos de exclusion social.

\section{Summary}

In this article, we analyzed the methodology of learning-communities from an inclusive education perspective. This is a qualitative study with a hermeneutical approach, in which we carried out an analysis of contents of textbooks, scientific articles, press articles, laws, curriculums, training programs and institutional norms. The results certify that the learning-communities methodology plays an important role in the inclusive education, because it takes into account: dialogue and communicative action, dialogic learning, dialogic model, interactive groups and literary gatherings, which allows the improvement of coexistence, capacity building, dialogue and criticism for the construction of an egalitarian, multicultural and solidarity society. Therefore, learning-communities help to overcome inequalities and the risk of social exclusion, as well as the student's tendency to fail.

Keywords: Learning communities; inclusive education; dialogic learning; inequalities; risks of social exclusion.

\section{Introducción}

T a filosofía de Comunidades de aprendizaje se fundamenta en dar respuesta a la escuela nueva, donde se Lcrean expectativas positivas en los estudiantes y se fomenta la participación; todo ello bajo un concepto de educación integradora, puesto que se involucran a los diversos actores de la comunidad participativa, porque se considera que la educación comienza en la calle, en la escuela y en todos los ámbitos donde nos movemos en la sociedad y permanentemente, porque continuamente estamos recibiendo información, que para procesarla debemos poseer herramientas para procesarla. Es por ello, que la transformación edu-

\footnotetext{
1 Candidata a Doctora en Educación, Profesora de la Universidad Pedagógica Nacional Francisco José Morazan. email: mlanza@upnfm.edu.hn

2 Doctor en Educación, Profesor, Investigador y Gestor de proyectos de la Universidad de las Regiones Autónomas de la Costa Caribe Nicaragüense, email: william.flores@uraccan.edu.ni
} 
cativa que se propone a través de las comunidades de aprendizaje conduce a la posibilidad de superar el fracaso escolar y mejorar la convivencia (Elboj, Puigdellívol, Soler, \& Valls, 2002).

En este sentido, se pretende analizar el enfoque de comunidades de aprendizajes desde una perspectiva de la educación inclusiva considerando: el diálogo y la acción comunicativa; aprendizaje dialógico, modelo dialógico, grupos interactivos y tertulias literarias, como herramientas fundamentales para una educación inclusiva.

\section{Revisión de la literatura}

Las experiencias de comunidades de aprendizaje se han desarrollado en países como España, Chile y Brasil. "En el campo de la Educación, las familias y las comunidades quieren participar en el diseño y desarrollo del centro y la educación que quieren para sus hijos" (Aubert, Flecha, García, Flecha, \& Racionero, 2009, p. 33). Para superar las desigualdades sociales los autores analizan que existe una fuerte relación entre los procesos dialógicos y el cambio social, a su vez se analiza como el aprendizaje tiene lugar en diferentes contextos y con la influencia de factores como es el género, la clase social o la etnia. Además, se toma como referente a Vygotsky destacando la importancia de la interacción social para el aprendizaje.

En 1978, tuvo lugar la primera comunidad de aprendizaje, el centro de educación de personas adultas de la Verneda-Sant Martí, a raíz de ello el centro de investigación de teorías y prácticas superadoras de desigualdades (CREA) de la Universidad de Barcelona, realiza investigaciones con el propósito de encontrar la forma de desarrollar esa experiencia de éxito, con lo que surgió el modelo de comunidades de aprendizaje, asesorando en 1995 la transformación en comunidades de aprendizaje de 3 centros en Euskadi y que en la actualidad existen 61 centros en España, 3 en Brasil y 2 en Chile (Gómez, Mello, Santa Cruz, \& Sordé, 2010). Se trata de un proyecto que se encamina a brindar una educación de calidad en funcion de los retos de la sociedad de la información y con base en las necesidades transformadas en los sueños de la escuela que se quiere, se priorizan y se les da seguimiento a través de grupos de trabajo conformados por los miembros de la comunidad educativa.

El convencimiento del claustro del CEP Ruperto Medina de Portugalete (Bilbao), promovió al CREA a iniciar el primer proceso de transformación de un centro educativo de educación infantil y primaria. Se formó un gran equipo, el Equipo de Comunidades de Aprendizaje, que posibilitó concretar las concepciones mencionadas en la introducción en un proyecto de transformación en comunidad de aprendizaje. Pero este proyecto, tal y como hoy se da, es también el resultado de la participación de todas las personas que han ido interviniendo en él: los claustros de los cuatro primeros centros de Euskadi (Ruperto Medina, Artatse, Virgen del Carmen y Ramón Bajo), sus asesores, la Dirección General de Renovación Pedagógica del Gobierno Vasco, y muchas otras personas y colectivos que han hecho real una esperanza que ya se está extendiendo por otros muchos centros. En la actualidad, las comunidades de aprendizaje ya son diez en Euskadi (Jaussi \& Garre, 2002) y más de una veintena en toda España (concretamente, en Euskadi, Aragón y Cataluña), donde abarcan desde la educación infantil hasta la educación secundaria (Racionero \& Serradell, 2005).

En relación a lo anterior, el único proyecto integrado del área de Ciencias Sociales, Económicas y Humanas del VI Programa Marco, coordinado por un centro de investigación español es el proyecto INCLUD-ED del Centro de Investigación en Teorías y Prácticas Superadoras de Desigualdades (CREA), de la Universidad de Barcelona, el cual se centra en el estudio de las interacciones entre los sistemas educativos, los agentes y las políticas, hasta el nivel obligatorio: infantil, primaria, secundaria y los programas de formación profesional y de educación especial (Valls, 2014). La investigación en el proyecto se desarrolló a través de la metodología comunicativa, que es un enfoque ideal para el trabajo investigador con grupos vulnerables, ya que el conocimiento se construye a través del diálogo entre los investigadores con su experiencia, así como los avances científicos reconocidos por la comunidad científica internacional y los distintos actores sociales, que aportan sus experiencias personales (Valls, 2014).

El equipo investigador del proyecto Includ-ED (Strategies for inclusion and social cohesion in Europe from education) ha realizado estudios de casos en las comunidades que desarrollan proyectos de aprendizaje donde integran intervenciones sociales y educativas que contribuyen a reducir las desigualdades y que para ello implican a las familias y la comunidad para contribuir al éxito académico del alumnado (Martínez \& Niemela, 2010). En 
cuanto los hallazgos de estos estudios se pueden mencionar que a mayor participación de las familias (Se habla de familiares y no de padres y madres, porque hay mucha diversidad entre las personas que conviven con las niñas y niños, en las escuelas), los niveles de alfabetización del alumnado mejoran, ya que ellos pueden ayudar a sus hijas e hijos en la realización de deberes y otras tareas escolares, mejorando los hábitos de estudio y la motivación a la educación (Valls, 2014).

La formación de familiares también tiene gran impacto en sus propias vidas, mejorando su autoestima y sus posibilidades para acceder al mercado laboral. Asimismo, la participación de las familias, en la toma de decisiones ha contribuido a la baja de los niveles de absentismo, también es posible llegar a acuerdos con el profesorado, en cuanto al tipo de educación que se quiere para las niñas y los niños, mediante el diálogo igualitario y así lograr las mejoras previamente descritas (Martínez \& Niemela, 2010).

\section{Materiales y métodos}

El presente estudio se enmarca en el paradigma cualitativo, porque es una actividad sistemática orientada a la comprensión en profundidad de fenómenos educativos y sociales, a la transformación de prácticas y escenarios socioeducativos, a la toma de decisiones y también hacia el descubrimiento y desarrollo de un cuerpo organizado de conocimiento (Bisquerra, 2009). Desde esta perspectiva, Hernández et al. (2010) explican que en el paradigma cualitativo el enfoque o diseño "es el abordaje general que utilizará en el proceso de investigación (p. 492). En este sentido, está investigación tiene un enfoque hermenéutico, porque se interpretaron los significados de contenidos de libros de textos, artículos científicos, artículos de prensa, leyes, currículos, programas de formación y normas institucionales.

Por tales razones, se utilizó como método el análisis de contenido, el cual ayudó a describir los documentos en sus partes esenciales. Para ello, se empleó la técnica de revisión de documento, donde nos centramos con los argumentos, las razones, los propósitos, los significados que los documentos presentan sobre comunidades de aprendizajes desde una perspectiva de la educación inclusiva. Por consiguiente, se construyó una guía de revisión documental como instrumento.

\section{Resultados y discusión}

\section{El diálogo y la acción comunicativa}

El diálogo, la aceptación de las aportaciones de los saberes de todas las personas, la transformación, el aprendizaje científico de las materias, la solidaridad, la aportación a partir de la realidad de cada grupo y la decisión y la reflexión sobre la propia vida son puntos importantes de la práctica educativa (Valls, 200o). En consecuencia, las teorías y modelos de aprendizaje como el aprendizaje dialógico, lo que a su vez tiene sus orígenes en la teoría de la acción comunicativa desarrollada por Habermas (2010), él que plantea que el lenguaje desempeña aparte de una función de entendimiento, la de coordinar las acciones teleológicas de los diversos sujetos de acción y que se desarrolla en sociedad; además, plantea que el individuo debe su identidad personal a la identificación e interiorización de las características de la identidad colectiva en interacción, es decir, que los niño y niñas al entrar en contacto con personas de referencia interiorizan las orientaciones valorativas de su grupo social y adquiere capacidades realizadas de acción.

La concepción comunicativa, aclara con respecto a los conocimientos previos y el aprendizaje significativo propuesto por la concepción constructivista, que estos se construyen y se transforman de manera individual y colectiva al mismo tiempo, a través de las interacciones (Jaussi \& Garre, 2002). En cuanto, a la función de entendimiento, la acción comunicativa sirve a la tradición y renovación del saber cultural.

\section{Aprendizaje dialógico}

La interacción social y el contexto donde se desarrolla, son el origen de la persona, asimismo las actitudes y pensamientos de la persona son el resultado de un proceso de desarrollo social mediatizado por el lenguaje (Flecha, 2009). Es decir, nuestro alumnado es el resultado de las interacciones que tienen con todas las personas con las que se relacionan en la escuela o el instituto y universidad, pero también de las relaciones que tienen con agentes educativos de contextos no escolares. El caso de las ciencias del aprendizaje no es diferente, y en las últimas décadas se ha producido un cambio hacia concepciones más comunicativas, que ha facilitado que los procesos de aprendizaje se estudien de forma preferente en el contexto de las 
interacciones sociales en las que éstas tienen lugar (Aubert, García, \& Racionero, 2009).

Cuando en los centros educativos actuamos dialogando con todos los agentes educativos con los que se relaciona nuestro alumnado, nuestras acciones serán más efectivas y completas. Para ello, resulta fundamentalmente, no sólo que demos voz a toda esa diversidad de personas, sino también que los diálogos que mantengamos con ellas estén basados en la igualdad. Eso es posible desde interacciones dialógicas y las pretensiones no son suficientes (Flecha, 2009).

Para que se desarrolle un aprendizaje dialógico, se requieren los siguientes principios: un diálogo igualitario entre las personas participantes, donde las aportaciones son valoradas en función de la validez de los argumentos y no en función de las relaciones de poder o jerarquías; inteligencia cultural que incluye la inteligencia académica, comunicativa y la práctica, que permite llegar a acuerdos en diferentes ámbitos sociales; transformación en el que se posibilitan los cambios en las propias personas y su contexto; dimensión instrumental, es decir incluir todos los conocimientos imprescindibles para vivir en la sociedad; creación de sentido, significa favorecer un aprendizaje que parte de la interacción, las demandas y necesidades de las propias personas, siendo éstas las que guíen su proceso de aprendizaje; solidaridad en la que se han de basar las prácticas educativas, cuyo propósito es la superación del fracaso escolar y la exclusión social; e igualdad de diferencias: es decir, valorar la diversidad de personas como un elemento de riqueza cultural, pero siempre junto con el valor de la igualdad (Aubert, García, \& Racionero, 2009).

Aubert, García, y Racionero (2009) nos dicen que; "los centros educativos con más nivel de aprendizaje y mejor convivencia son aquellos que abren sus espacios al diálogo igualitario entre profesorado, familiares, personas profesionales, voluntariado, alumnado y otras personas del entorno del alumnado" (p.132). En las comunidades de aprendizaje esto se observa en diferentes espacios y actividades. Uno de ellos es la organización escolar. Con la transformación del centro escolar en comunidad de aprendizaje se crean comisiones mixtas de trabajo formadas por el profesorado, alumnado, familiares, agentes locales, asociaciones. En las que juntos y a través del diálogo deciden qué acciones llevar a cabo, para conseguir los objetivos que toda la comunidad ha acordado, con el fin de aumentar los aprendizajes de todo el alumnado y mejorar la convivencia (Flecha, 2009).

\section{Modelo dialógico}

En el modelo dialógico, el concepto de educación que orienta implica una transformación desde las propias construcciones intersubjetivas de quienes comprenden la educación como un proceso que les involucra protagónicamente, y que no es sólo responsabilidad de la comunidad educativa, sino de toda la comunidad, hasta las acciones más cotidianas del trabajo en el aula y fuera de ella, donde toda la comunidad participa colaborando solidariamente (Ferrada \& Flecha, 2008). Desde esta construcción, se asume que la persona humana está dotada de la acción, por lo tanto, es agente-actuante, constructora y transformadora del medio en el cual se desarrolla y vive. Se sostiene que la formación de la persona cuenta con dos mecanismos de regulación que la constituyen como unidad. El primero, es el centro autopoético, que constituye al ser humano como una totalidad orgánica, el propio mecanismo regulador, que la hace humana en la interacción con el entorno, modificando su propia fisiología a la vez que modifica el lenguaje. El segundo, es el centro intersubjetivo, que se regula desde su lenguaje y acción, dotándola de actitud crítica, resultado de las interacciones que mantiene con sus personas de referencia, que a su vez la dotan de responsabilidad en sus actos y en el uso del lenguaje (Ferrada \& Flecha, 2008).

Desde esta perspectiva el tipo de sociedad que se maneja, es una donde existe la transmisión de la cultura que ha sido seleccionada para tales efectos; la integración social de los estudiantes a la comunidad a la que pertenecen, que se logra a través del conjunto de normas y reglas que rigen en ella; y la socialización, en cuanto a la formación de la personalidad del alumnado (Ferrada \& Flecha, 2008).

El currículum dentro de un modelo dialógico se define como una construcción social que surge de las interacciones de las personas que constituyen una comunidad educativa contextualizada histórica y socialmente, y que están sujetas tanto a relaciones de poder, como de diálogo. Es la propia comunidad, que responde a la pregunta ¿qué enseñar? y es ella que participa en esta construcción social, considerando la totalidad del contexto, tanto el local como el global (Ferrada \& Flecha, 2008). Desde esta conceptualización de currículo, es de suma importancia la selección de contenidos, que requiere de una construcción y validación colectiva, que constituye la base para transformar el fracaso en éxito escolar. En ello, es fundamental la participación del profesorado, el alumnado, los 
familiares, el vecindario y cualquier otro ciudadano que desee participar en la escuela, de manera que se transformen las desigualdades de aprendizaje del alumnado en aprendizajes máximos para todos y especialmente para aquellos colectivos en riesgo de exclusión social (Ferrada \& Flecha, 2008).

El modelo de aprendizaje asumido por todas las escuelas que se han transformado en Comunidades de Aprendizaje, tiene como característica esencial la promoción y generación de una igualdad de aprendizaje en todo el alumnado, para lo cual es necesario las siguientes claves para que se de ese aprendizaje. El diálogo igualitario, el cual se produce cuando todas las personas participantes profesionales de la educación, familiares, administradores, participantes en asociaciones y entidades del barrio, estudiantes participan en un plano de igualdad, donde los aportes de cada uno son importantes, según los argumentos presentados, no según la mayoría o según una imposición de poder, derivada, por ejemplo, de la jerarquía dentro de la estructura escolar (Ferrada \& Flecha, 2008).

La Inteligencia cultural, es decir la capacidad de aprender a través del diálogo y de las habilidades comunicativas que tienen las personas, esta constituye una capacidad universal, así todas las personas tienen inteligencia cultural, porque tienen capacidades innatas para comunicarse y actuar gracias al lenguaje. Asimismo, la inteligencia cultural va más allá de la inteligencia académica, significa el reconocimiento de la capacidad de aprendizaje y de la inteligencia de todas las personas, de cualquier cultura, barrio, etnia o edad (Ferrada \& Flecha, 2008). La transformación, para superar el fracaso escolar, se hace necesario un cambio en el contexto sociocultural del cual provienen los niños y niñas, es decir, poner en práctica todos los recursos para que cada persona llegue al máximo de sus posibilidades de aprendizaje, superar el dictamen como etiqueta paralizadora y modificarlo en instrumento de transformación de una situación personal o social (Ferrada \& Flecha, 2008).

La dimensión instrumental, son los conocimientos científicos, técnicos y el desarrollo de habilidades instrumentales necesarias para actuar con éxito en la sociedad, la selección de estos aprendizajes requiere de la participación del estudiantado y de sus familiares (Ferrada \& Flecha, 2008). Creación de sentido, vivimos en una sociedad abierta a una diversidad creciente de estilos de vida, la posibilidad de elegir el proyecto vital requiere de tener acceso a un abanico de posibilidades que puedan ofrecer la educación. Así, los niños y niñas no restringen sus aspiraciones, en cuanto a su desarrollo profesional a reproducir los mismos empleos de sus familias, sino que el conocer otras posibilidades y el confiar en la educación para lograrlo, implica la construcción de sentido para este alumnado y a elegir libremente (Ferrada \& Flecha, 2008).

La solidaridad, es un valor que se consigue sólo a través de la práctica solidaria, como un valor que se pone en práctica en las interacciones en el aula y, en primer lugar, por parte del profesorado. También es compartir procesos de aprendizaje en vez de separar al alumnado de forma competitiva, entre aquellos que están más capacitados, más interesados o más motivados, de otros que se colocan en grupos de muy bajo nivel y que coinciden pertenecer a los grupos más vulnerables socialmente (Ferrada \& Flecha, 2008). Igualdad de diferencias, sobre la base de la evidencia que la imposición de una igualdad homogeneizadora propicia la exclusión de los grupos minoritarios, el aprendizaje dialógico establece la igualdad de las diferencias afirmando que la verdadera igualdad respeta la diferencia, es decir, que todas las personas, desde su cultura, religión, género, estilo de vida, etc., tengan las mismas oportunidades a la vez que se fomenta la igualdad de resultados a través del éxito escolar, laboral y social (Ferrada \& Flecha, 2008).

Emocionalidad, se refiere al reconocimiento del otro desde su corporalidad hasta las relaciones y acciones sociales que establece con los demás, iniciándose en su reconocimiento en la emoción, es decir, que cada persona, en tanto, ser amorosamente biológico requiere ser reconocido como tal de forma previa y permanente a todo el proceso de aprendizaje. En cambio, la dimensión exclusora, omite esta característica básica de lo que significa el reconocimiento de lo humano (Ferrada \& Flecha, 2008).

El modelo didáctico, en términos metodológicos, es posible construir un modelo didáctico de carácter eminentemente interactivo, que basa su accionar en las múltiples interacciones de la mayor diversidad de agentes sociales que sea posible ingresar al aula, a fin de ofrecer la mayor diversidad de formas de enseñar y formas de aprender. Esta metodología incorpora agentes sociales, que realizan un trabajo voluntario de colaboración en el aprendizaje, de preferencia al interior del aula, pero también en otros espacios de aprendizaje en el centro escolar, como las bibliotecas o las aulas de informática, bajo la orientación del profesor responsable. Los colaboradores de aprendizaje, son 
adultos provenientes de la comunidad cercana a la escuela, de la sociedad en general, o niños de los propios cursos, como también de cursos superiores de la propia escuela que manifiestan interés por colaborar con sus compañeros. Los colaboradores de aprendizaje, que constituyen un grupo muy heterogéneo a nivel cultural, lingüístico, religioso, educativo, de género y exclusión social (Ferrada \& Flecha, 2008).

El rol del profesorado, radicalmente pasa de ser un agente solitario y único moderador del proceso de enseñanza y aprendizaje, a ser un coordinador profesional de un equipo de colaboradores de aprendizaje que ponen en práctica una diversidad de oportunidades de aprendizaje para el conjunto de sus estudiantes (Ferrada \& Flecha, 2008). En cuanto al rol del alumnado, pasa de ser un agente pasivo receptor de conocimiento y de normativas establecidas, a ser protagonista de la creación dialógica de conocimiento. De tener un comportamiento de masa, pasa a tener un comportamiento de comunidad sin estar categorizado por rangos de rendimiento académico y rotulado por diagnósticos clínicos, sino ser considerado en condiciones de igualdad con respeto de su propia diferencia, además, no visualizado como un alumnado de bajas expectativas, sino depositario de altas expectativas académicas y sociales (Ferrada \& Flecha, 2008).

La organización del aula, cambia radicalmente de la tradicional, se organiza, mayoritariamente, en lo que se conocen como grupos interactivos. Así, de trabajar con un aula organizada en filas de estudiantes, y donde sólo hay una persona adulta, se distribuye el alumnado en pequeños grupos, de número, dependiendo del tamaño del grupo-clase y de voluntarios en el aula, a cargo de un colaborador de aprendizaje, que rotan permanentemente, una vez finalizada cada actividad, para cambiarse al siguiente grupo, configurando un aula en permanente dinamismo. De un trabajo pedagógico que utiliza el lenguaje sólo para alcanzar un objetivo, al uso de un lenguaje que permite actualizar y renovar consensos, transmitir y compartir emociones y sentimientos, así como proponerse alcanzar un entendimiento racionalmente motivado (Ferrada \& Flecha, 2008).

Como resultado de la interacción entre diferentes personas, que aportan sus sentimientos, saberes, vivencias y experiencias se crea el conocimiento. Este conocimiento es orientado a través del diálogo con el propósito de alcanzar los mayores acuerdos posibles en función a un aspecto de la realidad, alrededor de los contenidos que se enseñan y sobre el mismo centro educativo (Aubert, Flecha, García, Flecha, \& Racionero, 2009).

\section{Grupos interactivos}

Se trabaja con grupos heterogéneos en una misma clase o en aulas diferentes, sin separación por niveles educativos, con los que se atiende la diversidad, lo importante es mantener la heterogeneidad, pero incrementando el número de adultos en el aula, es decir, organizando grupos inclusivos a través de docentes de apoyo, familiares y voluntariado. Así, todo el alumnado, sean cuales fueren sus dificultades, recibirán los mismos conocimientos y los apoyos necesarios para alcanzarlos (Valls, 2014; Ferrer, 2005). En las comunidades de aprendizaje, ingresan en las aulas miembros de las familias, personas adultas del barrio, voluntariado universitario, ex-alumnado, personal de asociaciones culturales, etc. Se trata de personas con rasgos muy diversos al del profesorado y con imaginarios muy diferentes del alumnado por lo que es posible transformar las interacciones educativas, que no sólo aumentan los aprendizajes instrumentales, sino que a su vez mejoran la convivencia e incrementan la solidaridad (Flecha, 2009). Un aspecto importante a considerar, es que los adultos participantes deben poseer altas expectativas de aprendizaje, principio que constituye un eje vertebral del proyecto de las comunidades de aprendizaje, por lo que se plantea la conveniencia de revisar, la actitud del profesorado y de los tutores y tutoras (Ferrer, 2005).

Para implementar los grupos interactivos, el alumnado del aula se divide en grupos heterogéneos de cuatro o cinco alumnos/as cada uno, asegurándose que posean mayores y menores habilidades en la materia o asignatura a trabajar, así como representantes de los diferentes grupos culturales que hay en el aula. El profesor o la profesora diseña tantas tareas como grupos haya en el aula y cada adulto es responsable de apoyar el desarrollo de una de las tareas. Las tareas tendrán una duración de unos 15 minutos, de tal modo que, en una sesión, cada grupo realice un mínimo de cuatro tareas, a la vez que se produce una interacción entre ellos y con los adultos (Valls, 2014). Por otra parte, esta estrategia, crea espacios de aprendizaje eficaces en un clima de aula basado en la comunicación y la cooperación, tanto de los estudiantes entre sí, y con las personas adultas responsables del seguimiento de los grupos. Las altas expectativas de aprendizaje, principio que constituye un eje vertebral del proyecto de las comunidades de aprendizaje, plantea la conveniencia de revisar, si es necesario, la actitud del profesorado y de los/las tutores/as en el marco de los grupos interactivos (Ferrer, 2005). 
En los grupos interactivos, la persona con discapacidad se beneficia al trabajar con el alumnado que aprende a un ritmo más rápido y con estándares de aprendizaje más exigentes. Además, contando con la ayuda de sus compañeros y compañeras, puede participar en las mismas actividades que el resto de la clase. Así, por una parte, se evita que el alumnado salga del aula de referencia para ir al aula de Educación Especial. Todo ello, redunda tanto en la mejora de su aprendizaje como en su integración social. La ayuda del propio voluntariado y de los compañeros puede ser innecesario el apoyo directo del profesorado de Educación Especial dentro del aula. Pero no es sólo el escolar con discapacidad el que se beneficia de su inclusión en el aula ordinaria. INCLUD-ED ha puesto de manifiesto que la inclusión también aporta beneficios al resto del alumnado (Valls, 2014).

\section{Tertulias literarias}

En algunas comunidades de aprendizaje se realizan tertulias literarias dialógicas. A través del diálogo igualitario, en los cuales se encuentran personas que nunca han leído un libro. La tertulia, se desarrolla poniendo en común párrafos que han llamado la atención o generan alguna reflexión en la lectura individual previa. Generalmente se realizan sesiones semanales en las que se comentan los capítulos que se acuerden del libro elegido, ya sea por un alumno o alumna o un adulto, se profundiza en el texto y no se hace necesario llegar a una interpretación de consenso. En las tertulias literarias dialógicas se mejoran todas las competencias lingüísticas y se estimula también que los participantes relacionen la lectura con experiencias personales por lo que se generan vínculos de amistad y cohesión más fuertes (Valls, 2014). En un proceso dialógico como el de comunidades de aprendizaje, la interacción entre las prácticas de éxito y las teorías educativas y sociales son construidas día a día por la diversidad de sectores participantes. Se construye tanto en el aula y el domicilio como en las tertulias donde se debate con el libro en la mano (Puigvert \& Santacruz, 2006).

\section{Conclusiones}

En esta investigación se ha analizado el enfoque de comunidades de aprendizajes desde una perspectiva de la educación inclusiva. El análisis permite decir que las comunidades de aprendizaje priorizan lo que pretenden conseguir; entre las acciones, se puede encontrar los grupos interactivos y la formación de familiares, para llevar a cabo esas prioridades y otras tareas, se crean comisiones de trabajo que facilitan la participación cotidiana en el centro y el aprendizaje.

En la construcción de las comunidades de aprendizajes hay que tomar en cuenta la etapa de sensibilización, toma de decisiones y el sueño. La sensibilización, es el análisis del contexto social en la que se encuentran y los conocimientos que requieren los niños y niñas y que en los próximos años trabajarán en ello. La toma de decisiones, consiste en decidir el inicio del proyecto con el compromiso de la comunidad educativa. Y el sueño, trata de uno de los lemas que debe guiar la participación del profesorado es: que el aprendizaje que queremos para nuestras hijas e hijos esté al alcance de todas las niñas y niños.

Algo importante a señalar sobre comunidades de aprendizajes, es un instrumento para la educación inclusiva porque contribuye a que: la escuela ejerce un papel primordial en la transformación del entorno; se fomenta la participación activa y crítica de todos los actores de la comunidad; se procura que la implicación de la comunidad sea realista, donde la metas son compartidas y los esfuerzos se encaminan hacia la misma dirección; la participación es activa tanto por el profesorado, las familias, el voluntariado que afectan las decisiones, la colaboración en el aula. El voluntariado tiene un gran valor dentro de las comunidades de aprendizajes y dependerá de la coincidencia de intereses; en comunidades de aprendizaje se crea un clima de altas expectativas para todos los actores de la comunidad educativa; se busca las estrategias necesarias para que todo el alumnado desarrolle al máximo sus capacidades y logre el aprendizaje que es el propósito central.

Las comunidades de aprendizajes permiten la superación de las desigualdades, es decir, se apuesta por la igualdad educativa reduciendo las situaciones de desigualdad y exclusión social, así como, la tendencia al fracaso del alumnado que pertenece a familias no académicas. Además, promueve una educación de calidad para todas las personas, es decir, el desarrollo de las competencias para enfrentarse a los retos de la sociedad de la información. También, permiten mejorar la convivencia, el desarrollo de capacidades de diálogo y crítica para una construcción de una sociedad igualitaria, multicultural y solidaria. 
En conclusión, en relación a la educación inclusiva esta debe enfocarse en la adquisición de habilidades básicas para acezar y seleccionar la información, el uso de las TIC, así como habilidades de comunicación y diálogo y no a la memorización. Otro aspecto, es que el enfoque tradicional de la enseñanza se centra en el individuo dejando de lado las prácticas sociales, es necesario, por lo tanto, que en la educación inclusiva se trabaje más de forma colaborativa con interacciones entre el alumnado más diverso, estos procesos que potencian las habilidades comunicativas se deben ver reflejados en el diseño de los currículos, así como en la organización de las aulas. Asimismo, la diversidad que existe en la sociedad es de beneficio para los centros educativos, esta diversidad va más allá de los conocimientos previos o niveles de competencia, esta incluye pluralidad de culturas, etnias, orígenes sociales, lenguas, genero, y familias.

\section{Bibliografía}

Aubert, A., Flecha, A., García, C., Flecha, R., \& Racionero, S. (2009). Aprendizaje Dialógico en la Sociedad de la Información. Barcelona: Hipatia.

Aubert, A., García, C., \& Racionero, S. (2009). El aprendizaje dialógico. Cultura y educación, 21(2), 129-139.

Bisquerra, R. (2009). Metodología de la Investigación Educativa. Madrid: La Muralla.

Elboj, C. (2005). Comunidades de Aprendizaje: Educar desde la igualdad de diferencias. Zaragoza: Gobierno de Aragón. Dpto. de Educación, Cultura y Deporte.

Elboj, C., Puigdellívol, I., Soler, M., \& Valls, R. (2002). Comunidades de Aprendizaje. Barcelona: Grao.

Ferrada, D., \& Flecha, R. (2008). El modelo dialógico de la pedagogía: un aporte desde las experiencias de comunidades de aprendizaje. Estudios pedagógicos, 34(1), 41-61.

Ferrer, G. (2005). Hacia la excelencia educativa en las comunidades de aprendizaje: participación, interactividad y aprendizaje. Educar (35), 61-70.

Flecha, R. (2009). Cambio, inclusión y calidad en las comunidades de aprendizaje. Cultura y Educación, 21(2), 157-169.
Flecha, R., \& Puigvert, L. (2004). las comunidades de aprendizaje: una apuesta por la igualdad educativa. Revista de estudios y experiencias en educación, 1(1), 11-20.

Gómez, A., Mello, R., Santa Cruz, I., \& Sordé, T. (2010). De las experiencias de comunidades de aprendizaje a las políticas basadas en su éxito. Interuniversitario de formación del profesorado, 67(24), 113-126.

Hernández, R., Fernández, C., \& Baptista, M. (2010). Metodología de la Investigación. México: McGraw-Hill.

Habermas, J. (2010). Teoría de la acción comunicativa. Madrid: Trotta.

Jaussi, M., \& Garre, C. (2002). Comunidades de Aprendizaje en Euskadi: una respuesta educativa en la sociedad de la información para todos $y$ todas. Servicio central de publicaciones del Gobierno vasco.

Krichesky, G., \& Murillo, F. (2010). Las comunidades profesionales de aprendizaje. Una estrategia de mejora para una nueva concepción de escuela. Iberoamericana sobre calidad, eficacia y cambio en educación, 9(1), 66-83.

Martínez, B., \& Niemela, R. (2010). Formas de implicación de familias y comunidad. Educación y pedagogía, 22(56), 69-78.

Racionero, S., \& Serradell, O. (2005). Antecedentes de las comunidades de aprendizaje. Educar, (35), 29-39.

Puigvert, L., \& Santacruz, I. (2006). La transformación de centros educativos en comunidades de aprendizaje. Calidad para todas y todos. Revista de Educación, 339, 169-176.

Valls, R. (2000). Comunidades de Aprendizaje. Una práctica educativa de aprendizaje dialógico para la sociedad de la información. Universidad de Barcelona. Barcelona.

Valls, R. (2012-2014). Actuaciones socioeducativas de éxito para la superación de la pobreza. Plan Nacional $I+D+I$. Secretaría de Estado de Universidades e Investigación. Ministerio de Educación y Ciencia. 